\title{
Newsfronts
}

\section{'Test-Tube' Mice Behaving Badly}

Mice produced using assisted reproductive technologies (ART) show long-term alterations in behavior, specifically related to anxiety and spatial learning. The possibility that the same long-term effects may ultimately apply to humans raises questions about the techniques commonly used to help infertile couples conceive.

About $1 \%$ of babies born in the Western world are conceived using ART, such as in vitro fertilization (IVF), in which oocytes are fertilized in vitro, cultured for a short time, and then returned to the womb. Since Louise Brown, the world's first 'test-tube' baby, was born in 1978, the use of ART has resulted in the conception of more than one million children. Retrospective studies have shown that 'test-tube' babies have a higher incidence of diseases such as Angelman and Beckwith-Wiedemann syndromes, which result from the loss of gene imprinting during embryonic development. There have as yet been no studies of long-term behavioral consequences of ART.

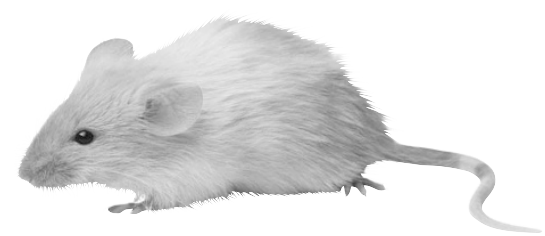

Now, Richard M. Schultz and colleagues at the University of Pennsylvania (Philadelphia, PA) present the results of a study in which they compared behavior in 'test-tube' mice with those conceived in vivo. Using the elevated zero maze to test anxiety and the Morris water maze to measure spatial memory, Schultz's team found that adults derived from cultured embryos were bolder and less able to retain spatial information than their control littermates (Proc. Natl. Acad. Sci. USA, 10 February).

The researchers speculate that these behavioral differences may be explained by slight changes in placental function, but they admit that it is not known whether the mouse is a good model for studying ART in humans. Nevertheless, despite the current trend toward culturing IVF embryos for longer periods, to allow doctors to pick the 'best' ones and to decrease the chances of multiple pregnancies, the authors point out that these results suggest "a special effort should be made to minimize the effect of culture on the human preimplantation embryo." Optimizing culture conditions may help to negate any potential effects on the developing embryo.

$$
\text { -Tanja Schub }
$$

\section{Is It Love ... or Addiction?}

The neurological mechanisms responsible for the 'reward' aspects of sex and drugs may also be responsible for the reinforcement of pair bonding in monogamous species, according to a new study from Emory University.

Numerous studies have identified the neural peptides oxytocin (OT) and arginine vasopressin (AVP) as potentially playing an important role in social pairing, and comparative analyses across monogamous mammalian species have strongly suggested that the action of these compounds in the ventral striatopallidal region of the brain may be particularly important.

In an effort to better define the physiological boundaries of OT and AVP action in the brain and the manner in which this localization might relate to the reinforcement of partnering behavior, Miranda Lim and her colleagues conducted a series of receptor-labeling and immunocytochemistry studies on brain sections from the prairie vole (Microtus ochrogaster), a popular animal model for the study of

\section{Cambridge University Nixes NHP Facility Plans}

Citing financial reasons, Cambridge University (UK) has canceled plans to build a stateof-the-art nonhuman primate neuroscience research facility. Professor Tony Minson, ProVice-Chancellor for Cambridge University, explained: "This has not been an easy decision to reach, but ultimately, we have a responsibility to our students and staff to take financial risks of this magnitude, and we believe that, although regrettable, this is the right course of action."

The proposed research facility would have provided a venue for conducting invasive neuroscience experiments aimed at preventing and treating brain ailments, including strokes and Alzheimer's and Parkinson's diseases; planners expected it to attract scientists from around the world. UK Prime Minister Tony Blair had expressed support for the project.

However, since it was first proposed in 2001, the project has drawn intense criticism from animal rights activists. In part as a result of planning delays and the need for extra security around the site, the costs of building the center had soared from an original esti-

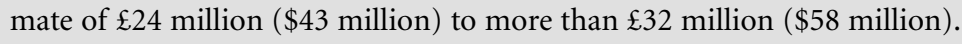

Cambridge's Minson asserts: "The animal rights groups will of course claim this as a victory, but in our view they have won no arguments whatsoever." The activists disagree. In a joint statement, Andrew Tyler of Animal Aid and Jan Creamer of the National AntiVivisection Society claimed: "This decision is an acknowledgment that Cambridge failed ... to demonstrate that these proposed experiments would be of any benefit to people."

The Cambridge decision has incensed many UK researchers. The Association of the British Pharmaceutical Industry's Director General Trevor Jones declared: "It is quite intolerable that much-needed research into conditions such as Alzheimer's and Parkinson's should be prevented from taking place by people who use intimidation, harassment and violence," in a statement issued on 27 January. 
monogamous pairing behaviors (J. Comp. Neurol., 19 January).

Their findings indicated that the AVP receptor $\mathrm{V} 1 \mathrm{aR}$ is primarily localized to the ventral pallidum, a brain structure that has been linked with reward-seeking motor behaviors. They found the OT receptor, OTR, primarily in the nucleus accumbens, a region heavily innervated by dopaminergic neurons that are thought to play a critical role in mediating 'reward' effects for a number of drugs of abuse and for natural positive stimuli such as sex and food.

The researchers observed some sex-specific differences; even though they found equivalent concentrations of OTR and $\mathrm{V} 1 \mathrm{aR}$ in males and females, the presence of AVP in the ventral pallidum appears to be specific to males. This is not surprising, according to Lim, since OT/OTR seems to be involved in maternal behavior patterns, whereas the AVP/V1aR system is "mostly specific to male-typical behaviors". These new data suggest the possibility of parallel, possibly sex-specific pathways that could work in conjunction with dopaminergic signaling to establish positive associations and reward partnering in animals with monogamous tendencies.

Lim also indicates that these findings could have some bearing on understanding the basis of other social ties not related to mating. "There are a lot of parallels to other forms of bonding," she tells $L a b$ Animal, "such as in mother-infant bond formation. A good animal model is in sheep who selectively bond with one lamb, and this behavior is mediated by OT/OTR in the brain." Several studies also indicate that alterations in the OT and AVP pathways play a role in the dysfunctional social bonding seen in autism.

Having identified the structures in the brain that are potentially involved in mediating these behaviors, Lim's group is now looking more closely at the mechanisms behind these pathways. "We have moved on to more functional studies," she says, "including pharmacological manipulation as well as manipulating OTR and V1aR expression using viral vector gene transfer in both monogamous and promiscuous vole species—a sort of 'gene therapy', if you will."

—Michael Eisenstein

\section{Creating Transgenics from Modified Germ Cells}

A new technique for the genetic modification of zebrafish sperm cells could be the first step in dramatically improving the efficiency of transgenic production.

Even as transgenic technology becomes an increasingly essential tool in biological research, there is still great interest in refining existing techniques to improve the speed and efficiency with which genetically modified organisms can be generated. Conventional transgenic techniques tend to result in so-called mosaic animals; only a limited number of embryos will exhibit the modifications in their germ cells that will allow them to pass these changes to their offspring, and this poses an important obstacle to the rapid production of transgenic lines.

Researchers from Fukui Prefectural University (Obama, Japan) and the National Human Genome Research Institute (Bethesda, MD) recently published (Proc. Natl. Acad. Sci. USA, 3 February) the results from their efforts to develop a technique for generating transgenic zebrafish through direct modification of the germ cells. Noriyoshi Sakai and his colleagues used a modified Moloney murine leukemia retrovirus to infect isolated zebrafish spermatogonia, which were cultured on a bed of testicular-derived feeder cells.

The mature sperm produced only 104 embryos from 1,111 eggs, of which only 5 were transgenic; however, all 5 showed mendelian transmission of the provirus. The authors suggest that this technique could lead to considerably improved efficiency and reduced husbandry requirements in the generation of transgenic fish, and could prove a powerful tool if adapted for use in mammalian models.

$-M . E$ 